\title{
Contrast sensitivity and glare in cataract using the Pelli-Robson chart
}

\author{
T H Williamson, N P Strong, J Sparrow, R K Aggarwal, R Harrad
}

\begin{abstract}
There is a need for a convenient, clinically applicable test of glare disability which can be used in the preoperative evaluation of patients with cataract. In this study, contrast sensitivity (using the Pelli-Robson letter chart), near vision, and visual acuity were compared, with and without the introduction of a glare source in 70 patients with cataract, 15 with intraocular lenses, and 19 controls. A disposable pen torch was shone at the pupillary margin to induce glare. Contrast sensitivity demonstrated the most marked reduction during glare testing. Cortical cataracts were most affected followed by posterior subcapsular opacities. The glare disability was significantly less in pseudophakic patients and was absent from patients with non-cataractous phakic eyes. Glare testing with a disposable pen torch and a Pelli-Robson contrast sensitivity letter chart provides a rapid test of glare disability which can be easily incorporated into the clinical appraisal of patients with cataract.

(Brf Ophthalmol 1992; 76: 719-722).
\end{abstract}

A discrepancy is often encountered between the visual function measured by Snellen acuity and the visual disability that is experienced by patients with cataract.' The disparity has become more apparent as the reliability, acceptability, and success rate of cataract extraction and intraocular lens implantation has improved. A measurement of visual disability by Snellen acuity alone is now less than satisfactory and additional tests of visual deficit and postoperative improvement are required.

Contrast sensitivity and glare disability are sensitive measurements of visual loss in patients with cataract, particularly those with mild or moderate lens opacities. Investigators using computer based monitor systems ${ }^{1-3}$ or projection devices $^{4}$ have demonstrated a reduction in contrast sensitivity in these patients. Paulsson and Sjostrand ${ }^{3}$ also tested the effect of glare on contrast sensitivity by placing a bright light source adjacent to a VDU displaying sinusoidal gratings and Abrahamsson and Sjostrand have used a TV monitor surrounded by a fluorescent tube for this purpose. ${ }^{56}$ These tests are sensitive measures of visual function but they are laborious to perform. The incorporation of these testing methods into routine practice would require the purchase of expensive equipment. If a test is to be used regularly in the clinic it is essential that it is quick, reproducible, and readily available. In this study, we report the results obtained by using a Pelli-Robson letter based chart to test contrast sensitivity and a disposable pen torch as the glare source. This test is simple and rapid, and could be easily introduced into a busy clinical environment.

\section{Patients and methods}

Subjects with cataract were recruited from patients attending the Department of Ophthalmology at Addenbrooke's Hospital, Cambridge. Patients with high myopia or ocular disease other than cataract were excluded.

All subjects were questioned as to the extent of their symptoms for near vision, distance vision, and glare disability and asked to rank these on a six point scale.

Snellen visual acuity was tested using a standard back illuminated Snellen chart at $6 \mathrm{~m}$. The appropriate spectacle correction was worn and the visual acuity was rounded off to the nearest line.

Jaeger plates were used to test near vision at $0.3 \mathrm{~m}$ with a reading correction.

Contrast sensitivity was tested with a PelliRobson chart. ${ }^{78}$ This chart consists of letters of constant size arranged in 16 groups of three. The contrast of all the letters in the first of these triplets is $100 \%$. The contrast of each subsequent triplet is reduced by a factor of $0.707(0.15 \mathrm{log}$ unit). Thus the contrast of the last triplet is $0.56 \%(2.25 \log$ units below $100 \%)$. The chart was used at $1 \mathrm{~m}$ at a mean luminance of $65 \mathrm{~cd} / \mathrm{m}^{2}$ as recommended by the suppliers.

Glare was introduced with a disposable pen torch which was held at 20 degrees to the visual axis at $30 \mathrm{~cm}$ from the eye and directed at the pupil. At this distance the pen torch has a mean luminance of $4000 \mathrm{~cd} / \mathrm{m}^{2}$. Snellen acuity, Jaeger near acuity, and Pelli-Robson contrast sensitivity were tested without and then with the pen torch glare. The pen torch was replaced after every five patients. The pens were tested with a photometer which confirmed that there was no loss of brightness with this amount of usage.

The lens opacity of each cataract patient was graded for type (posterior subcapsular, nuclear, cortical, or mixed) and for severity (on a three point scale).

\section{STATISTICAL METHODS}

For the purpose of statistical analysis the Snellen and Jaeger acuities were converted to equivalent value of visual angle using the decimal scale in which $6 / 6$ has the value of $1 \cdot 0,6 / 12$ the value $0 \cdot 5$, $6 / 60$ the value $0 \cdot 1$, and so on. The effect of glare upon vision for near (J-diff) or distance (VA-diff) was expressed as the difference between the 
logarithms of the values obtained with and without glare. As the Pelli-Robson chart gives a logarithmic measure of contrast sensitivity the effect of glare upon contrast sensitivity (CS-diff) was obtained directly by subtraction.

A multivariate analysis using Rosner's intraclass correlation model was performed. This is the most efficient statistical method for the analysis of studies with a two eye design and continuous dependent variables. The method avoids the overestimation of significance levels which occurs if individual eyes are regarded as independent units of observation. The analysis was performed using the Generalised Linear Interactive Modelling (GLIM) system of the Numerical Algorithms Group (Wilkinson House, Jordan Hill Road, Oxford, OX2 8DR). In the analyses eyes were classified by lens status into groups and subgroups according to cataract type (cortical, posterior subcapsular, nuclear, and mixed), pseudophakia, and phakic noncataractous controls.

\section{Results}

\section{PATIENT CHARACTERISTICS}

The mean age of the group with cataract was $72 \cdot 8$ years (SD 9.4, range 44-91); of the pseudophakic patients was 72.9 years (SD 9.4, range 64-86); and of the non-cataractous phakic controls was $60 \cdot 9$ years (SD 10.0, range $44-76$ ).

In the cataract group there were 105 eyes in 70 patients; in the pseudophakic group there were 15 eyes in 15 patients; and in the control group with clear lenses there were 34 eyes in 19 patients. The 105 eyes with cataract were further divided into subgroups by cataract morphology such that there were 17 eyes with cortical cataract, 28 eyes with posterior subcapsular cataract, 37 eyes with nuclear cataract, and 23 eyes with mixed cataract morphology.

\section{SNELLEN VISUAL ACUITY. JAEGER VISION AND CONTRAST SENSITIVITY}

There were no significant differences between the visual acuities without glare in the four cataract subgroups $(p=0.09)$. Without glare, Snellen acuity correlated well with contrast sensitivity $(\mathrm{r}=0.74)$, Jaeger acuity correlated well with contrast sensitivity $(r=0.73)$, and Snellen acuity correlated well with Jaeger acuity $(r=0 \cdot 75)$. Despite these apparently good correlations between the various measures of visual function, there remains considerable spread about the regression line between these variables. This is exemplified graphically in Figure 1, where it can be seen that for a given Snellen acuity there remains quite a wide range of contrast sensitivity.

\section{GLARE EFFECT}

The pen torch glare effect was regarded as the difference in visual function measured with the glare source off and on. The effect of glare on visual function was examined in the groups with cataract, pseudophakia, and clear lenses, as well as in the four cataract subgroups.

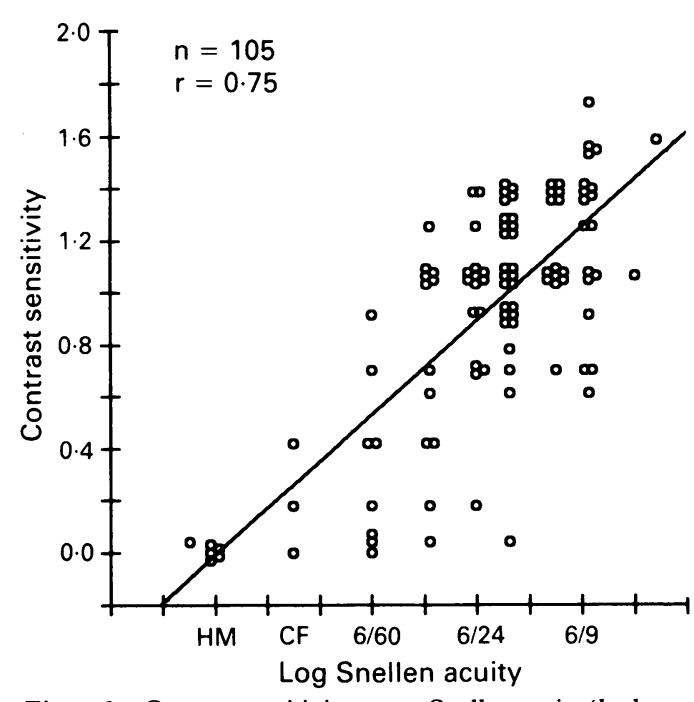

Figure 1 Contrast sensitivity versus Snellen acuity (both on a log scale) prior to surgery for all the patients in the study.

When the effect of glare upon contrast sensitivity was compared between the different types of cataract there were significant differences between these groups $(p=0.0025)$. The subtype of cataract producing the greatest loss of contrast in response to glare was the cortical, followed in reducing order by posterior subcapsular, mixed, and nuclear cataract.

There were no differences in the glare effect upon Snellen acuity between the cataract subgroups, pseudophakic, or control subjects $(p=0.34)$. The glare effect upon Jaeger vision, however, was not uniform across these groups $(p=0.045)$. The difference in performance was accounted for by the non-cataractous phakic controls, who were less affected by glare than the cataractous and pseudophakic subjects. Compared with the controls the other subgroups were affected from most to least in the order: posterior subcapsular cataract, mixed cataract, cortical cataract, nuclear cataract, and pseudophakia (Table 2). Differences within this rank ordering however were not significant $(p=0 \cdot 13)$.

There were marked differences in the glare effect between the groups and subgroups with regard to contrast sensitivity $\left(p<10^{-6}\right)$. This powerful effect was mainly due to differences between the non-cataractous phakic controls and the other groups. There was a significant difference between the pseudophakic subjects and the non-cataractous controls $(0.005)$, the pseudophakic subjects suffering a more marked loss of contrast sensitivity than the controls. The pseudophakic subjects experienced slightly less loss of contrast sensitivity than the subjects with cataracts (Table 3), though this effect was statistically non-significant $(p=0 \cdot 14)$.

\section{SYMPTOMS}

Snellen acuity and visual symptoms at distance showed a strong association $\left(\mathrm{p}<10^{-6}\right)$ and symptoms of reading difficulty correlated similarly with Jaeger vision $\left(\mathrm{p}<10^{-6}\right)$. However symptoms of glare difficulty were not significantly associated with the effect of glare upon Snellen acuity $(p=0 \cdot 20)$, Jaeger acuity $(p=0.71)$, or contrast sensitivity $(\mathrm{p}=0 \cdot 61)$. 
Table 1 The mean distance visual acuity for each of the groups, with and without glare, and the mean effect of glare. (All data on a linear scale)

\begin{tabular}{llll}
\hline Type & With glare & Without glare & Difference \\
\hline PSCLO* & 0.354 & 0.374 & 0.0196 \\
Nuclear & 0.326 & 0.340 & 0.0142 \\
Cortical & 0.431 & 0.436 & 0.0049 \\
Mixed & 0.276 & 0.292 & 0.0160 \\
IOL $\dagger$ & 0.606 & 0.630 & 0.0240 \\
Control & 0.887 & 0.887 & 0.0000 \\
\hline
\end{tabular}

$\star$ Posterior subcapsular lens opacity.

†Intraocular lens.

Table 2 The mean faeger near acuity for each of the groups, with and without glare, and the mean effect of glare. (All data on a linear scale)

\begin{tabular}{llll}
\hline Type & With glare & Without glare & Difference \\
\hline PSCLO & $0 \cdot 148$ & 0.200 & 0.0515 \\
Nuclear & 0.194 & 0.222 & 0.0275 \\
Cortical & $0 \cdot 221$ & 0.283 & 0.0620 \\
Mixed & 0.122 & 0.181 & 0.0590 \\
IOL & 0.306 & 0.329 & 0.0227 \\
Control & 0.354 & 0.354 & 0.0000 \\
\hline
\end{tabular}

Table 3 The contrast sensitivity for each of the groups, with and without glare, and the mean effect of glare

\begin{tabular}{llll}
\hline Type & With glare & Without glare & Difference \\
\hline PSCLO & 0.701 & 0.991 & 0.2896 \\
Nuclear & 0.690 & 0.861 & 0.1705 \\
Cortical & $0 \cdot 154$ & 0.660 & 0.4941 \\
Mixed & 0.560 & 0.777 & 0.2161 \\
IOL & 0.991 & $1 \cdot 143$ & 0.1519 \\
Control & 1.453 & 1.453 & 0.0000 \\
\hline
\end{tabular}

\section{Discussion}

As demonstrated by Figure 1, contrast sensitivity for a given visual acuity varies quite widely between patients. CS loss, therefore measures a different visual disability compared with loss of Snellen acuity and can provide the clinician with added information concerning the extent of visual disability. ${ }^{1}$ This is especially the case in patients whose visual acuity remains good despite significant lens opacities. Contrast sensitivity drops as the severity of the cataract increases and improves markedly after cataract extraction and lens implantation. ${ }^{9}$

Patients with cataract often complain about glare - for example, from bright sunlight or car headlights, and may find this glare more disabling than a moderate drop in visual acuity. These symptoms have been termed glare disability ${ }^{10}$ and are due to increased intraocular light scatter and subsequent loss of contrast of the retinal image. Attempts have been made to provide clinically applicable tests of glare. For instance Prager $e t$ al measured Snellen acuity under office light compared with that in sunlight as a measure of glare" and Maltzman used a pen torch to provide glare whilst measuring Snellen visual acuity. ${ }^{12}$ However Snellen acuity is little affected by loss of contrast so these tests can be expected to be insensitive. Our finding of a much more marked effect of glare upon CS than either Snellen acuity or Jaeger near vision suggests that CS is a substantially more sensitive measure of glare disability than the other two tests.

We did not find a correlation between the subjective symptoms of visual glare and the measured effect of glare upon contrast sensitivity. This may reflect the difficulty of devising a meaningful scoring system for subjective glare disability. Different patients may have rated the same degree of glare disability quite differently.

All forms of cataract showed glare disability. In keeping with common clinical experience posterior subcapsular lens opacities showed severe glare loss. However the marked extent to which glare also affected the patients with cortical lens opacities was unexpected.

After cataract extraction and intraocular lens implantation the glare disability is much reduced but an effect of glare is still demonstrable. ${ }^{13-15}$ In the present study the patients with extracapsular cataract extraction and posterior chamber lens implantation had a mean reduction in CS owing to glare of 0.152 (Table 3) yet the control group was found to have no loss owing to glare at all. This glare disability in pseudophakia may be attributable to light scattering either by the posterior capsule or by the intraocular lens.

The advantages of contrast sensitivity and glare disability measurements in the clinical setting can be illustrated by the following case. A 23-year-old male, who worked as a labourer, presented complaining of difficulty seeing in bright light. He wore sunglasses when he worked outdoors but these brought only partial benefit and he was unable to read a number plate at 25 yards in daylight and thus unable to drive. $\mathrm{He}$ was found to have congenital cataracts, more marked in the right eye, and had already undergone a right lensectomy 3 years previously. Unfortunately, the visual acuity in this eye improved only to $6 / 36$ with contact lens correction because of the presence of stimulation deprivation amblyopia. The visual acuity in the left eye was $6 / 6$ with appropriate refractive correction but the contrast sensitivity on the Pelli-Robson chart was only 0.80 (normal range $1.65-1.89$ for a 20-30-year-old). This became unrecordable in the presence of the glare light. A snowstorm punctate type of congenital cataract was present in the left eye.

Many surgeons would have been reluctant to carry out a cataract extraction and lens implant on this patient in view of the good visual acuity. However accurate measurements of the visual deficit by contrast sensitivity and glare disability strongly supported the decision to operate. The patient therefore underwent cataract extraction and lens implantation and subsequently a YAG capsulotomy. The postoperative visual acuity was $6 / 6$ and the contrast sensitivity improved to 1.65 and did not deteriorate with glare. The patient is now able to work without any difficulty and is planning to take his driving test.

This study shows that the Pelli-Robson chart is a much more sensitive means of demonstrating glare disability than Snellen or Jaeger acuity. Using the chart and a pen torch it is possible to show in patients with cataract that contrast sensitivity is reduced by glare to a much greater extent than in normal controls. The Pelli-Robson chart is both convenient and quick to use. It is easy for the patient to perform and the observer to interpret. In this study it was found to be a useful clinical test of contrast sensitivity and glare disability in patients with cataract. This test may prove particularly useful in those patients whose complaints are out of proportion to their Snellen acuity. 
1 Hess R, Woo G. Vision through cataracts. Invest Ophthalmol Vis Sci 1978; 17: 428-35.

2 Elliot DB, Gilchrist J, Hurst $M$, Pickwell LD, Sheridan $M$ Weatherill J. The subjective assessment of cataract Ophthalmol Physiol Opt 1989; 9: 16-9.

3 Paulsson LE, Sjostrand J. Contrast sensitivity in the presence of glare light. Invest Ophthalmol Vis Sci 1980; 19: 401-6.

4 Hirsch RP, Nadler MP, Miller D, Glare measurements as a predictor of outdoor vision among cataract patients. Am f Ophthalmol 1984; 16 : $965-8$.

5 Abrahamsson $M$, Sjostrand J. Impairment of contras sensitivity function (CSF) as a measure of disability glare. sensitivity function (CSF) as a measure of
Invest Ophthalmol Vis Sci 1986; 27: 1131-6.

6 Sjostrand J, Abrahamsson M, Hard AL. Glare disability as a cause of deterioration of vision in cataract patients. Acta Cause of deterioration of vision in cataract

7 Pelli DG, Robson JG, Wilkins AJ. The design of a new letter chart for measuring contrast sensitivity. Clin Vis Sci 1988; 2: 187-9.

8 Zhang L, Pelli DG, Robson JG. The effects of luminance, distance and defocus on contrast sensitivity as measured by the Pelli-Robson chart. Invest Ophthalmol Vis Sci 1990; 30 Suppl): 406

9 Adamsons I, Rubin GS, Abbey H, Stark WJ. Correlation of visual questionnaire results with glare and contrast tes results in cataract patients. Invest Ophthalmol Vis Sci 1992, 33: 1301

10 Hirsch RP, Nadler MP, Miller D. Clinical performance of a disability glare tester. Arch Ophthalmol 1984; 102: 1633-6.

11 Prager TC, Urso RG, Holladay JT, Stewart RH. Glare testing in cataract patients: instrument evaluation and identification of sources of methodological error. 7 Cataract Refract Surg 1989 ; 15: 149-57.

12 Maltzman BA, Horan C, Rengel A. Penlight test for glare disability of cataracts. Ophthalmic Surg 1988; 19: 356-8.

13 Nadler DJ, Jaffe NS, Clayman HM, Jaffe MS, Luscombe SM Glare disability in eyes with intraocular lenses. Am $\mathscr{f}$ Glare disability in eyes
Ophthalmol $1984 ; 97: 43-7$.

14 Weatherhill J, Yap M. Contrast sensitivity in pseudophaki and aphakia. Ophthalmol Physiol Opt 1986; 6: 297-301.

15 Koch DD, Jardeleza TL, Emery JM, Franklin D. Glare following posterior chamber intraocular lens implantation. f Cataract Refract Surg 1987; 13: 431-5.

更 